\title{
Bulk Mode Disk Resonator With Transverse Piezoelectric Actuation and Electrostatic Tuning
}

\author{
Mohannad Y. Elsayed, Student Member, IEEE, Paul-Vahé Cicek, Member, IEEE, \\ Frederic Nabki, Member, IEEE, and Mourad N. El-Gamal
}

\begin{abstract}
This paper presents a wine-glass bulk mode disk resonator based on a novel transverse piezoelectric actuation technique to achieve bulk mode resonance of the single crystalline silicon disk structure. The device is fabricated in a commercial microelectromechnical systems (MEMS) process, and combines reasonable quality factor and superior motional resistance in a low-cost technology. External capacitive electrodes are used for electrostatic tuning of the resonance frequency, relying on the electrostatic spring softening effect. The resonator was measured to have a resonance frequency of $\sim 15 \mathrm{MHz}$ and a quality factor of $\sim 2000$ in atmospheric pressure, increasing to $\sim 5000$ in 100 -mtorr vacuum. The temperature co-efficient for the frequency of the device was also measured to be about $-40 \mathrm{ppm} /{ }^{\circ} \mathrm{C}$. The resonator requires no dc voltage for operation, but its resonance frequency can be tuned by varying the applied dc voltage on the capacitive electrodes with a factor of $1 \mathrm{ppb} / \mathrm{V}^{2}$.

[2015-0202]
\end{abstract}

Index Terms-Microelectromechanical systems (MEMS), micromachined resonators, bulk mode resonators, piezoelectric actuation, aluminum nitride (AIN), bulk micromachining, PiezoMUMPs, timing.

\section{INTRODUCTION}

$\mathbf{M}$ ICROMACHINED resonators are receiving continuously increasing attention due to their small sizes compared to conventional crystal-based resonators as well as their potential for integration with other MEMS resonators and circuits on the same chip (e.g., [1]), which can be especially advantageous for handheld electronic applications (e.g., smartphones and tablets) where weight, size, and cost are particularly critical parameters.

Micromachined resonators can be operated through two main widespread actuation mechanisms: piezoelectric or electrostatic, each method having its specific advantages and drawbacks. Piezoelectric devices such as surface acoustic wave (SAW) and film bulk acoustic resonators (FBAR) are already widely used in timing applications [2]. Piezoelectric

Manuscript received July 16, 2015; revised December 15, 2015; accepted December 18, 2015. This work was supported in part by McGill University, in part by the Natural Sciences and Engineering Research Council of Canada, in part by the Quebec Fund for Research in Nature and Technology, and in part by the Microsystems Strategic Alliance of Quebec. Subject Editor G. Piazza.

M. Y. Elsayed, P.-V. Cicek, and M. N. El-Gamal are with the Department of Electrical and Computer Engineering, McGill University, Montreal, QC H3A 0E9, Canada (e-mail: mohannad.elsayed@mail.mcgill.ca; paul-vahe.cicek@mail.mcgill.ca; mourad.el-gamal@mcgill.ca).

F. Nabki is with the CoFaMic Research Center, Université du Québec à Montréal, Montreal, QC H3C 3P8, Canada (e-mail: nabki.frederic@uqam.ca).

Color versions of one or more of the figures in this paper are available online at http://ieeexplore.ieee.org.

Digital Object Identifier 10.1109/JMEMS.2016.2514267
FBARs generally exhibit high electromechanical transduction efficiencies and low signal transmission losses, resulting in low motional resistances, which is very advantageous as it simplifies the design constraints of the associated electronic circuitry and results in lower power consumption. Also, piezoelectric devices do not require any DC voltage for operation. However, conventional piezoelectric FBAR devices generally suffer from lower quality factors, notably limited by the resonator-to-electrode strain loss at the interface [3], [4], and their resonance frequencies are limited by the piezoelectric layer thickness, as they utilize out-of-plane vibration modes. This limitation makes it impractical to achieve more than one resonant frequency on the same chip [5]. Lateral mode devices (e.g., [5]-[8]) use in-plane modes, and can achieve different resonant frequencies by adjusting the device dimensions. In [6], integrated nanoscale heating elements are used for temperature control of aluminum nitride MEMS contour mode resonators. The heating elements are separated from the resonators structures by sub-micron air gaps in order to maintain the electromechanical properties of the devices. In [7], integrated switches serve to create a reconfigurable contour mode resonator. In [8], another type of lateral vibrating piezoelectric resonators is presented based on lithium-niobate. It is to be noted that piezoelectric actuation does not provide inherent means for frequency tuning.

As for electrostatic resonators, they rely mostly on vibratory resonance, either in a flexural or bulk mode. Bulk-mode devices typically exhibit high stiffness, and are consequently less prone to thermoelastic damping, compared to flexural devices, allowing them to achieve large quality factors $(>10,000)$, even at atmospheric pressure [9]-[18]. However, electrostatic actuation causes these resonators to exhibit lower electromechanical transduction efficiency and significant signal transmission loss, leading to higher motional resistance than typical piezoelectric devices. This can however be mitigated by increasing the applied DC polarization voltage and utilizing sophisticated technologies to realize very thin lateral transduction gaps $(<100 \mathrm{~nm})$ (e.g., [9]-[18]). Electrostatic resonators can also benefit from the electrostatic spring softening phenomenon to allow for tuning the resonance frequency by varying the polarization voltage. This effect cannot be replicated for typical piezoelectric devices. In [10]-[13], capacitive bulk-mode disk resonators exhibiting quality factors of 2,000-150,000 and motional resistances of $1.5 \mathrm{k}-1 \mathrm{M} \Omega$ using polarization voltages $<20 \mathrm{~V}$ are demonstrated. In [3], actuation and sensing of a bulk-mode 
disk resonator made of AlN is performed by a combination of piezoelectric and capacitive methods. The device resonates at $51 \mathrm{MHz}$ with quality factor of $\sim 13,000$ and a loss of $\sim-34 \mathrm{~dB}$. However, no tuning capability is reported. In [4], a radial mode AlN resonator is presented. It employs a capacitive-piezoelectric actuation scheme similar to [3], using metal electrodes above and beneath the disk structure. The electrostatic electrodes beneath the disk structure are also used to pull it down to the substrate and consequently power the resonator off upon the application of a switching DC voltage. Notably, all the aforementioned bulk-mode designs require complex fabrication process steps to realize submicron gaps in order to avoid excessive motional resistances. In [19], a flexural (out-of-plane) piezoelectric resonator is presented. The device employs a metal electrode beneath the suspended structure for electrostatic tuning. However, it operates at a relatively low frequency of $\sim 32 \mathrm{kHz}$ due to the low stiffness flexural mode of operation. In [20], a different method of frequency tuning is presented, where electromechanical feedback of the drive-mode displacement signal is used in order to achieve dynamic mode-matching of the proposed piezoelectric gyroscope. Electrostatic resonating bulk structures using commercial micromachining technologies were fabricated before [14]-[16]. In [14] and [15], the devices had to operate at high voltages $(>50 \mathrm{~V})$ in order to overcome the dimension limitations on the transducer gap imposed by the technology. In [17] and [18], combs were added to the bulk-resonating structure in order to improve overall sensitivity and allow for operation at lower voltage, at the expense of increased area.

Due to the limitations of the aforementioned designs, the motivation behind this work is to combine transverse piezoelectric actuation with bulk mode resonance in order to achieve low motional resistance without requiring any DC voltage, reasonable quality factor (even in air), and a relatively high resonance frequency. Notably, quality factors that are higher than FBARs can be achieved because the device uses a single metal electrode layer topping the resonator structure. This introduces less $Q$-limiting resonator-toelectrode strain loss compared to conventional FBAR designs that require additional metal electrodes beneath the structure. This is achieved without requiring any DC voltage for operation, and thus the design brings together the advantages of piezoelectric actuation and bulk-mode resonators. The work also presents optional electrostatic tuning of the device. Since proper operation of the resonator does not require narrow transduction gaps, it is fabricated in a relatively simple and low resolution technology (MEMSCAP PiezoMUMPs).

The paper begins with a description of the device design, followed by finite-elements simulation results. The process flow for the fabrication technology is then detailed, and measurement results are presented and discussed.

\section{DESIGN}

Figure 1 shows a simplified schematic of the proposed design. The device is composed of a single-crystalline silicon central disk structure acting as the wine-glass resonator. This disk is $10 \mu \mathrm{m}$ thick and has a $200 \mu \mathrm{m}$ diameter. It is covered by a $0.5 \mu \mathrm{m}$ layer of aluminum nitride, the piezoelectric

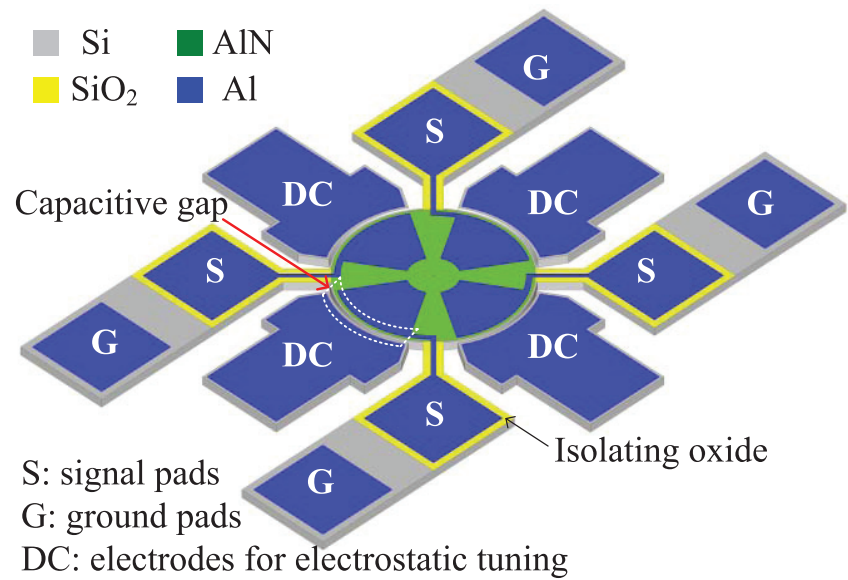

Fig. 1. Simplified diagram of the disk resonator outlining the pads used for piezoelectric actuation and electrostatic tuning.

material used for transduction and to bring the resonator into its wine-glass mode of resonance. The aluminum nitride covers the disk completely in one design, and in another it is patterned into four distinct quadrants. Both designs exhibit similar performance. The disk structure is supported by four suspension beams having a $10 \mu \mathrm{m}$ width (the minimum allowed by the design rules of the technology) with $90^{\circ}$ angular spacing, so as to correspond with the nodal points of the wine-glass resonance mode. The support beams are anchored to the substrate at their ends and mechanically connected to the electrical pads. Each of these supports is associated with a pair of pads, one for the signal routed above the piezoelectric layer, and the other for the ground, routed through the underlying silicon structural layer. For this purpose, an aluminum layer above the disk structure is patterned into four distinct quadrants, in order to match the strain distribution shown in Fig. 2. Each electrode is electrically connected to a distinct signal pad by an aluminum track routed above its respective suspension beam. The conductive structural silicon layer itself acts as the ground plane of the device, and connects with each ground pad while remaining electrically insulated from the signal track by a layer of silicon dioxide. Aluminum nitride is not present on the supports to avoid any unintended transduction which would alter the resonance mode and possibly lead to undesirable spurious modes of vibration. The disk structure is also surrounded by four capacitive electrodes which are separated from it by a lateral transduction gap of $2.5 \mu \mathrm{m}$, the critical spacing imposed by the fabrication process. These electrodes are intended for electrostatic tuning of the resonance frequency through the electrostatic spring softening effect, as later explained in section IV. Table I summarizes the design parameters of the resonator device fabricated here.

Finite-element simulations illustrating the mode-shape and the strain distribution of the resonator in the wine-glass mode are shown in Fig. 2. The resonance frequency of the disk in wine-glass mode is simulated to be of $17.54 \mathrm{MHz}$.

\section{Piezoelectric Actuation}

Operation of the proposed device relies on the piezoelectric effect, a relationship across the electrical 


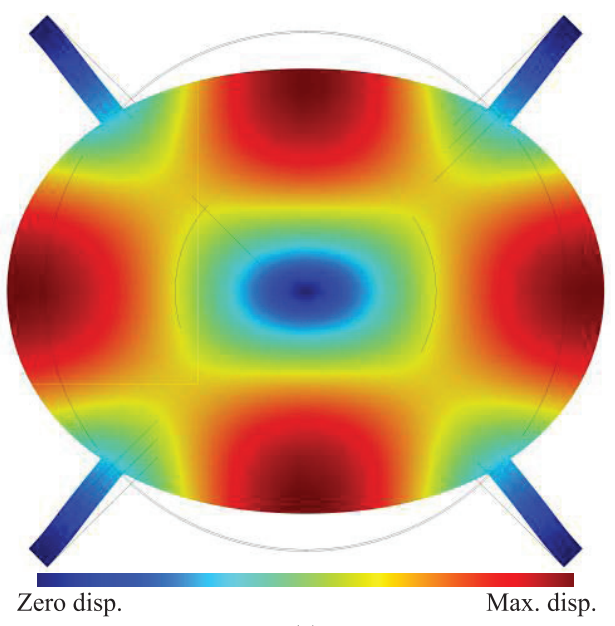

(a)

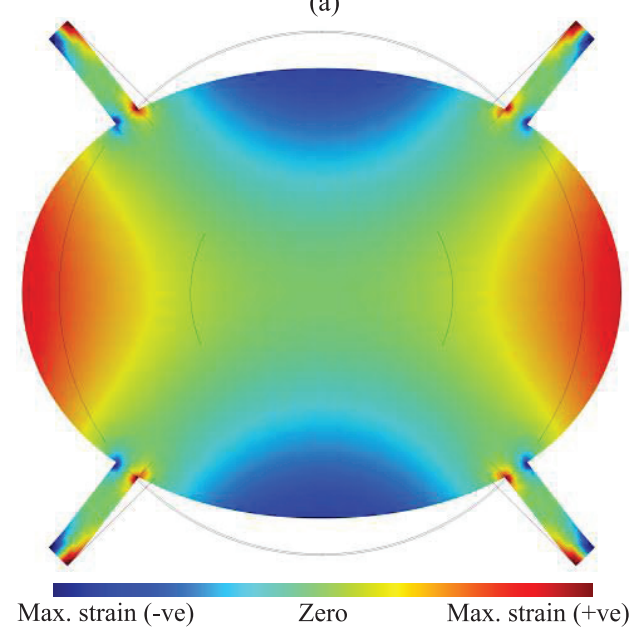

(b)

Fig. 2. (a) Wine-glass mode shape of the resonator and (b) its strain distribution.

TABLE I

Summary of Resonator Device Design Parameters

\begin{tabular}{l|c}
\hline \multicolumn{1}{c|}{ Parameter } & Value \\
\hline Disk diameter $(\mu \mathrm{m})$ & 200 \\
Structure thickness $(\mu \mathrm{m})$ & 10 \\
Support width $(\mu \mathrm{m})$ & 10 \\
Capacitive gap $(\mu \mathrm{m})$ & 2.5 \\
AlN layer thickness $(\mu \mathrm{m})$ & 0.5 \\
Simulated resonance frequency $(\mathrm{MHz})$ & $\sim 17$ \\
\hline
\end{tabular}

and mechanical domains. For certain materials with the proper atomic structure and crystal orientation, a mechanical stress results from electric charge accumulating across the material. This effect can also be used in reverse where a charge results from a mechanical stress. This relationship can be quantified, with the electric field and stress expressed as independent variables, by [21]

$$
\begin{aligned}
& D=\varepsilon \quad E+d \sigma, \\
& \xi=d^{T} E+S \sigma,
\end{aligned}
$$

where $D$ is the electric displacement vector, $\varepsilon$ is the dielectric constant matrix, $E$ is the electric field intensity vector, $d$ is the piezoelectric coefficient matrix, $\sigma$ is the stress tensor, $\xi$ is the strain tensor, and $S$ is the compliance matrix.

For this device, the vertical electric field resulting from the input voltage between the input electrodes on top of the disk and the ground plane is converted to an in-plane mechanical stress in the aluminum nitride layer through its transverse piezoelectric coefficient, $d_{31}$, based on

$$
\left|\sigma_{\text {in-plane }}\right|=\left|\frac{\varepsilon_{33} v_{\text {in }}}{d_{31} t}\right|,
$$

where $v_{\text {in }}$ is the input voltage, $\varepsilon_{33}$ is the vertical dielectric constant of the piezoelectric layer (AlN for this design), and $t$ is the piezoelectric layer thickness, i.e. the distance between the signal electrodes and the ground plane. The quadrant subdivision of the electrodes was selected for the induced stress pattern to force a mechanical actuation of the disk in the wine-glass mode shape. Based on its structural and dimensional properties, the disk filters this mechanical perturbation to favor strain at frequencies close to its resonance, in an identical fashion to usual electrostatically-actuated disk resonators. The reverse piezoelectric effect serves to convert this piezoelectrically-induced and mechanically-filtered strain back into an electrical output voltage based on (2).

\section{Electrostatic Tuning}

Generally, any resonator can be modeled as a spring-massdamper system. Its resonance frequency can be expressed as [21]

$$
f_{0}=\frac{1}{2 \pi} \sqrt{\frac{k_{e q .}}{m_{e q} .}},
$$

where $k_{e q}$. and $m_{e q}$. are the equivalent spring constant and mass of the structure respectively.

Based on a parallel-plate approximation, the capacitance between the electrodes and disk can be calculated as

$$
C \approx \frac{\varepsilon_{0} A}{g+x}
$$

where $\varepsilon_{0}$ is the free space permittivity, $A$ is the overlap area between the electrostatic electrodes and the disk, $g$ is the gap between the electrodes, and $x$ is the lumped displacement of the disk.

When a DC voltage, $V_{\text {tuning }}$, is applied between the tuning electrodes and the grounded disk, an electrostatic force $\left(F_{\text {elec. }}\right)$ is generated as given by [21]

$$
F_{\text {elec. }}=\frac{1}{2} \frac{d C}{d x} V^{2} \approx-\frac{\varepsilon_{0} A}{2(g+x)^{2}} V_{\text {tuning }}^{2} .
$$

This force impedes the motion of the resonator and therefore is equivalent to reducing the equivalent spring constant of the structure by a certain value, $k_{\text {elec. }}$, expressed as [22]

$$
k_{\text {elec. }}=-\frac{d F_{\text {elec. }}}{d x} \approx \frac{\varepsilon_{0} A}{g^{3}} V_{\text {tuning }}^{2}, \quad x \ll g .
$$


This spring softening effect alters the resonance frequency of the structure from its original value to

$$
\begin{aligned}
f_{r} & =\frac{1}{2 \pi} \sqrt{\frac{k_{e q .}-k_{\text {elec. }}}{m_{\text {eq. }}}}=f_{0} \sqrt{1-\frac{k_{\text {elec. }}}{k_{\text {eq. }} .}} \\
& =f_{0} \sqrt{1-\frac{\varepsilon_{0} A}{k_{\text {eq. }} g^{3}} V_{\text {tuning }}^{2} .}
\end{aligned}
$$

Such tuning is typically limited to capacitive-based resonators, as the piezoelectric devices do not readily allow for a similar softening effect. In this work however, the design presented makes use of this electrostatic tuning method in order to allow for frequency tuning of the resonance induced via piezoelectric actuation.

\section{FABRICATION PROCESS}

The resonators in this work were fabricated in the PiezoMUMPs technology from MEMSCAP. The fabrication process sequence, as detailed in [23], is illustrated in Fig. 3 for the devices fabricated here. The process begins with $150 \mathrm{~mm}$ n-type double-side polished silicon on insulator (SOI) wafers, as shown in Fig. 3(a). The top silicon surface is doped using phosphosilicate glass (PSG) in order to increase its conductivity. This layer constitutes the main structural layer that will be used to form the device, and that is connected to the electrical ground. A $200 \mathrm{~nm}$-thick thermal silicon dioxide is then grown, patterned and etched using reactive ion etching (RIE), as illustrated in Fig. 3(b). This thermal oxide serves to isolate the signal pads and interconnects from the ground plane in the areas without aluminum nitride (e.g., the signal pads or the top of supports). A $0.5 \mu \mathrm{m}$ thick layer of aluminum nitride is then deposited, patterned, and wet etched, as shown in Fig. 3(c). This piezoelectric layer is used for transduction. A metal stack composed of $20 \mathrm{~nm}$ of chrome and $1 \mu \mathrm{m}$ of aluminum is subsequently deposited and patterned through a lift-off process to form the electrical interconnects and pads, as illustrated in Fig. 3(d). At this stage, silicon is lithographically patterned, and etched using deep reactive ion etching (DRIE) to form the structural disk and the tuning electrodes, as shown in Fig. 3(e). Afterwards, a protective material is applied to the frontside of the substrate, to serve as a cover during subsequent backside etch steps. The substrate layer is then lithographically patterned and etched from the backside using DRIE to form release trenches that stop at the oxide layer. Wet oxide etch then removes the nowexposed buried oxide layer in the trench regions, as shown in Fig. 3(f). The frontside protection material is finally stripped by dry plasma etching, which completes the release of the disk structure and allows for its free motion, as illustrated in Fig. 3(g). Figure 4 shows an SEM micrograph of one of the fabricated devices.

\section{Measurement Results}

\section{A. Resonance Configurations and Characteristics}

The structure was intentionally designed with four independent signal electrodes to enable its use within both singleended and differential configurations, making it possible to
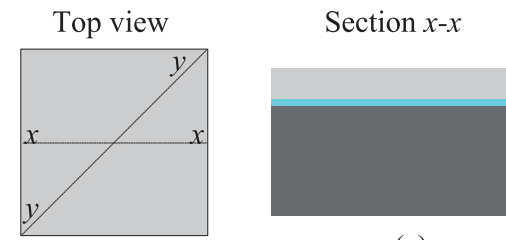

Section $y-y$
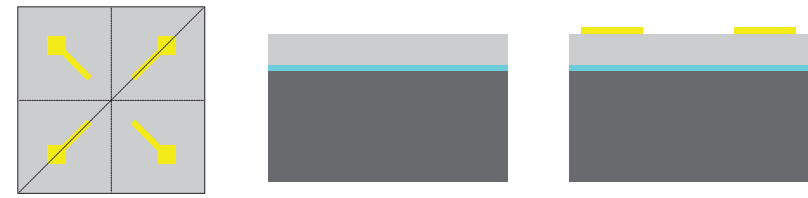

(b)
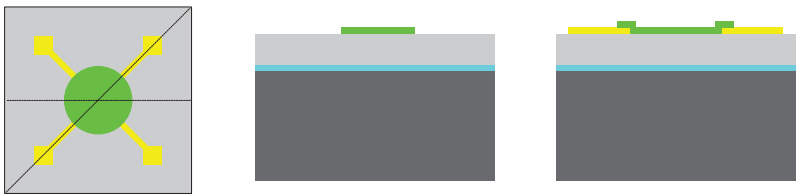

(c)
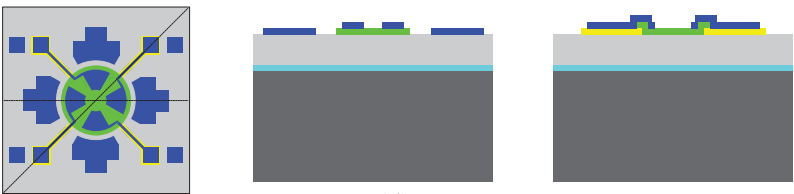

(d)
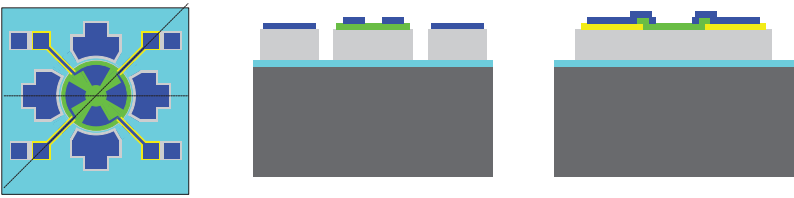

(e)
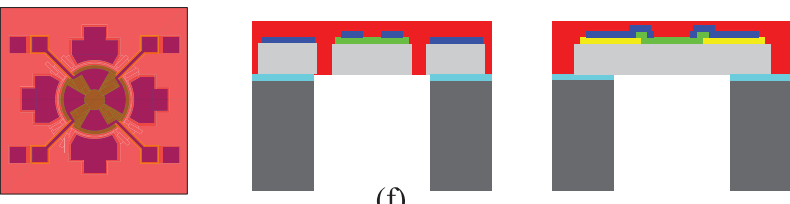

(f)
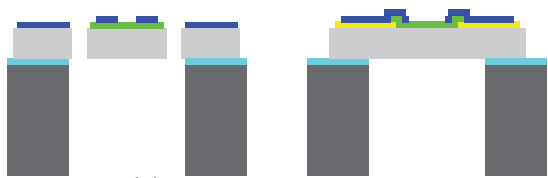

(g)

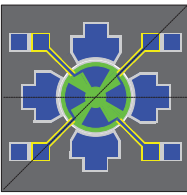

Buried $\mathrm{SiO}_{2}$

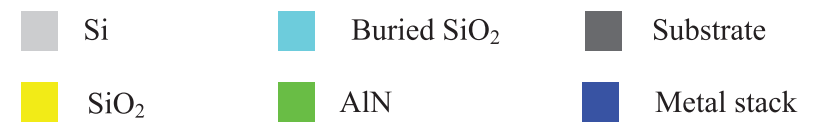

AlN

Metal stack

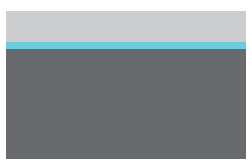

(a)

Protective layer

Fig. 3. Simplified PiezoMUMPs fabrication process flow.

directly connect to different amplifier types without the need for extra components or converters such as baluns. The dies of the tested devices were wire bonded directly onto a goldplated PCB, as shown in Fig. 5. This direct bonding approach was preferred to packaging in order to avoid added parasitics. 


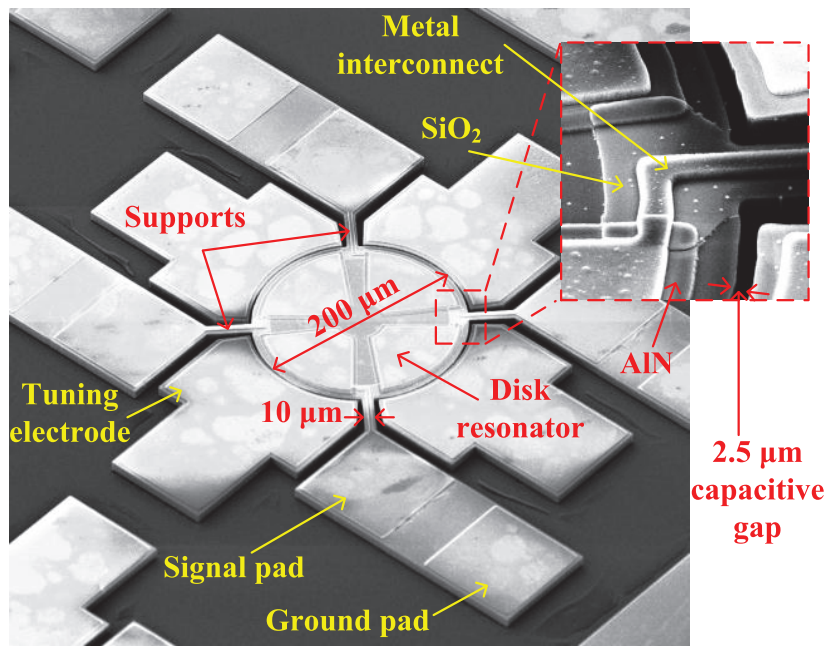

Fig. 4. SEM micrograph of one fabricated resonator.

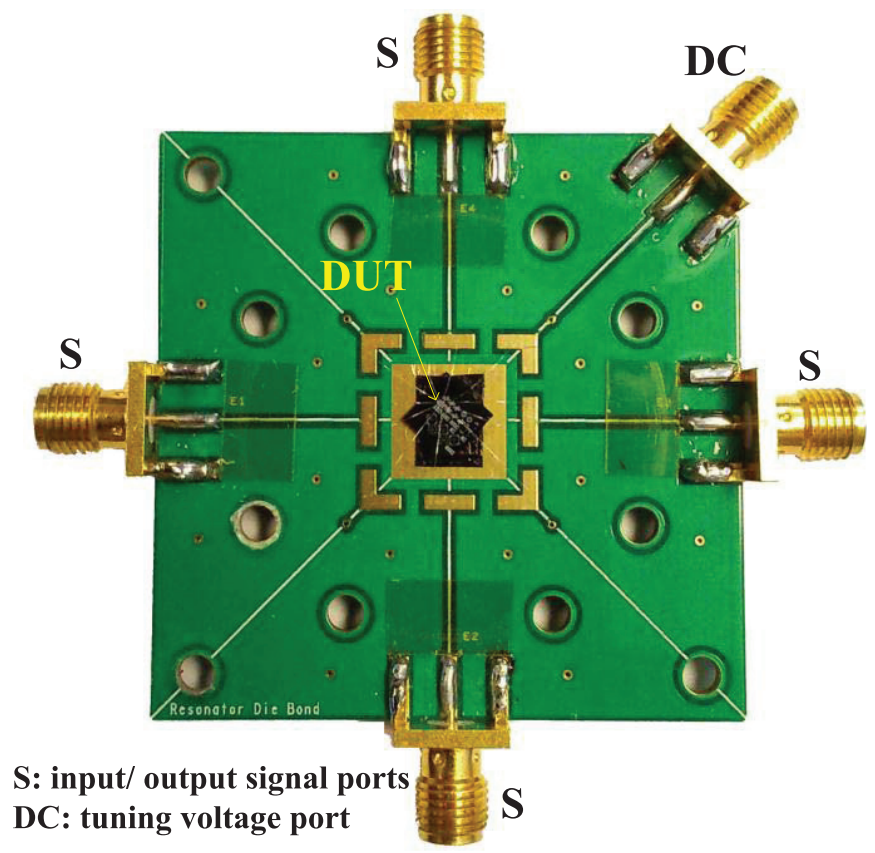

Fig. 5. Printed circuit board used for testing the resonator. A resonator die is directly wire bonded onto the board.

The resonance characteristics of the devices were measured using the test setups shown in Fig. 6, both at atmospheric pressure and in $\sim 100$ mTorr vacuum. Measurement results for the devices are shown in Fig. 7. The device exhibits a resonance frequency, $f_{r}$, of $14.63 \mathrm{MHz}$ and quality factor, $Q$, of $\sim 2,000$ and $\sim 4,900$ under atmospheric pressure and 100 mTorr vacuum level, respectively. The quality factor was extracted directly from the 3-dB bandwidth in the device transmission curves. Although modal analysis in simulations projected a resonance frequency of $17.54 \mathrm{MHz}$, the difference between the simulated and measured values can reasonably be attributed to the residual stress of the different layers, to the discrepancies between the structural material parameters used for simulation and their actual values, and to dimension

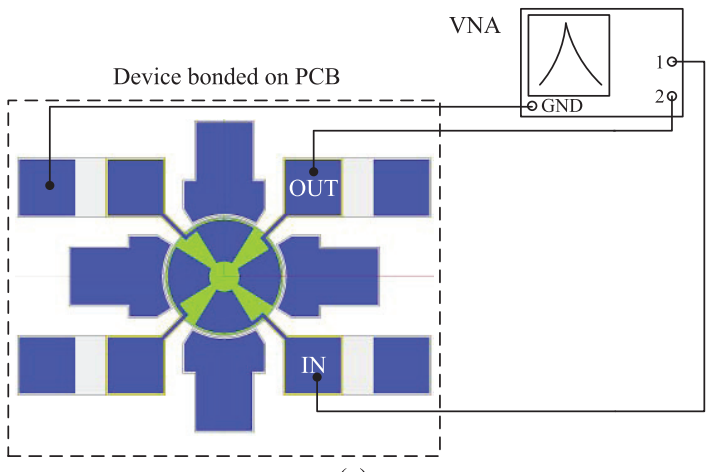

(a)

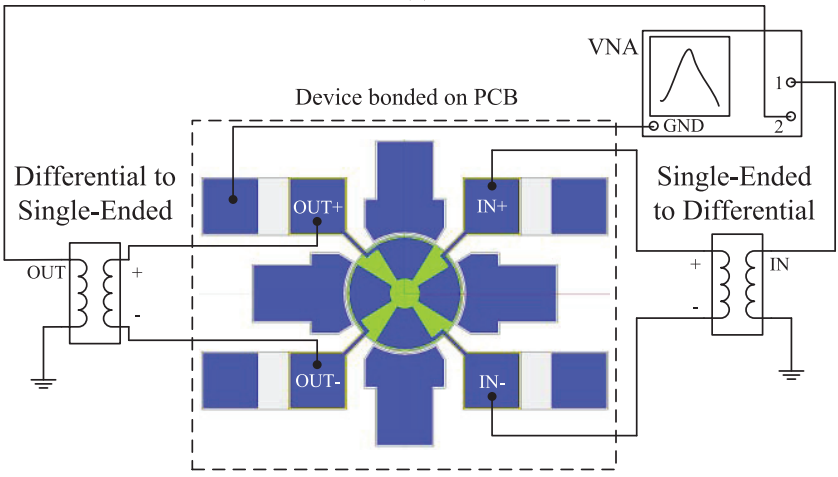

(b)

Fig. 6. Different resonance testing configurations: (a) single-ended, and (b) differential.

variations of the fabricated device. The resonator exhibits a transmission of $-32 \mathrm{~dB}$ (i.e., an insertion loss of $32 \mathrm{~dB}$ ) for the single-ended configuration in a 100 mTorr vacuum level, which corresponds to a motional resistance of $\sim 3.9 \mathrm{k} \Omega$. In the differential configuration, the transmission is higher at $-22.6 \mathrm{~dB}$ in vacuum, corresponding to a motional resistance of $\sim 1.2 \mathrm{k} \Omega$. This increase in transmission is expected because of the additional drive and sense electrodes used in the differential configuration. It is worth mentioning that simulation predicts an insertion loss of $\sim 12 \mathrm{~dB}$ in vacuum ( $20 \mathrm{~dB}$ lower than measurements). The reason for the discrepancy between simulation and measurements can be attributed to the difference between the material piezoelectric coefficients used for simulation and the actual ones.

Figure 8 illustrates the effect of the ambient pressure level on the resonance quality factor. Air damping has relatively little impact on the device performance, as the quality factor varies only by a factor of $\sim 2.5$ over the full range of the studied pressures. The quality factor approaches its maximum at pressures below 5 Torr. Two main effects were considered as potential causes for the limited quality factor measured, since the structural silicon layer is crystalline and its thermoelastic damping should not be a limiting factor, namely: i) thermoelastic damping from the top AIN layer, and ii) anchor loss. AlN damping was ruled out as the cause since two fabricated versions of the devices, one with AlN covering the disk completely, and the other with AlN patterned into four distinct quadrants under the electrodes, exhibit nearly the same quality factor despite the varying AlN disk area coverage. 


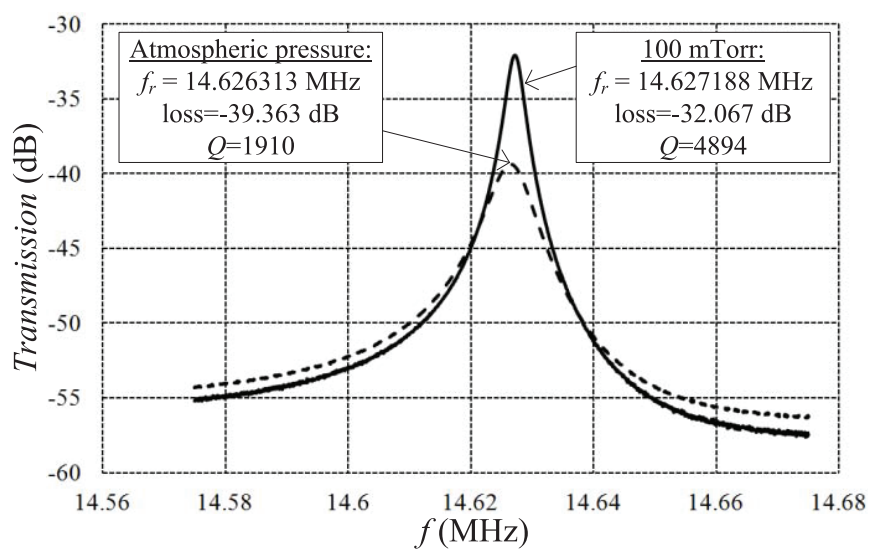

(a)

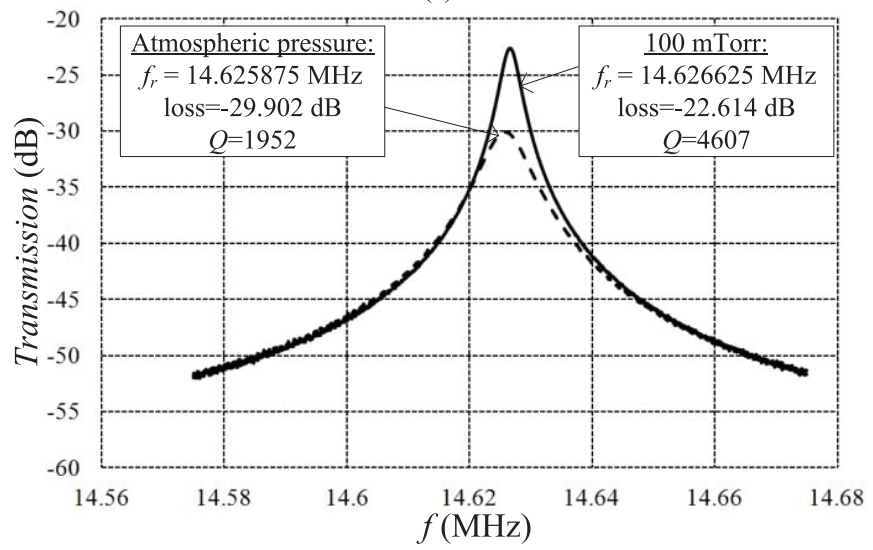

(b)

Fig. 7. Measured resonance characteristics of the resonators at atmospheric pressure (dashed line) and in vacuum (solid line) in (a) single-ended configuration, and (b) differential configuration.

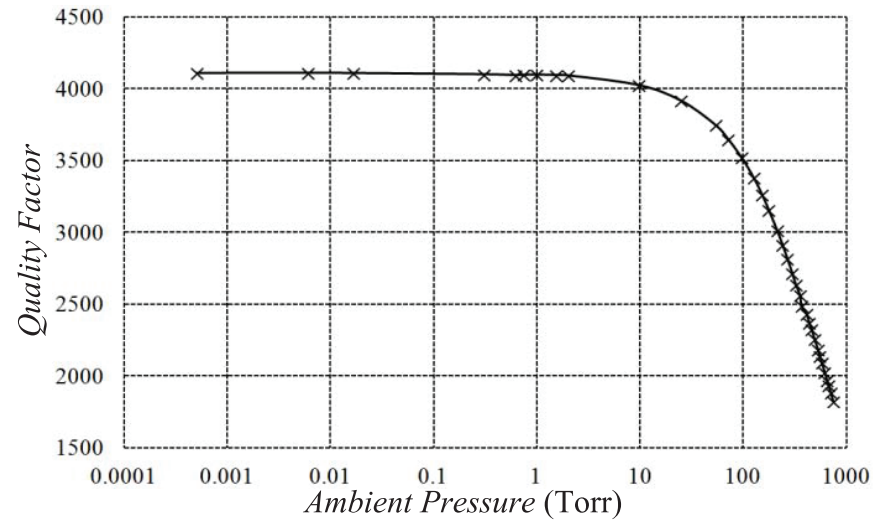

Fig. 8. Resonator quality factor vs ambient pressure.

This remains in-line with the results presented in [3], which confirmed that AlN is a high-Q material. The maximum quality factor is therefore likely limited by anchor loss through the supports. These supports are relatively wide due to fabrication technology constraints $(10 \mu \mathrm{m})$. However, the design would be capable of reaching higher quality factors if the supports could be made narrower, as noted in [3], where changing the supports by $1 \mu \mathrm{m}$, from $2 \mu \mathrm{m}$ to $1 \mu \mathrm{m}$, led to a quality factor increase of $\sim 80 \%$. Notably, the silicon supports of

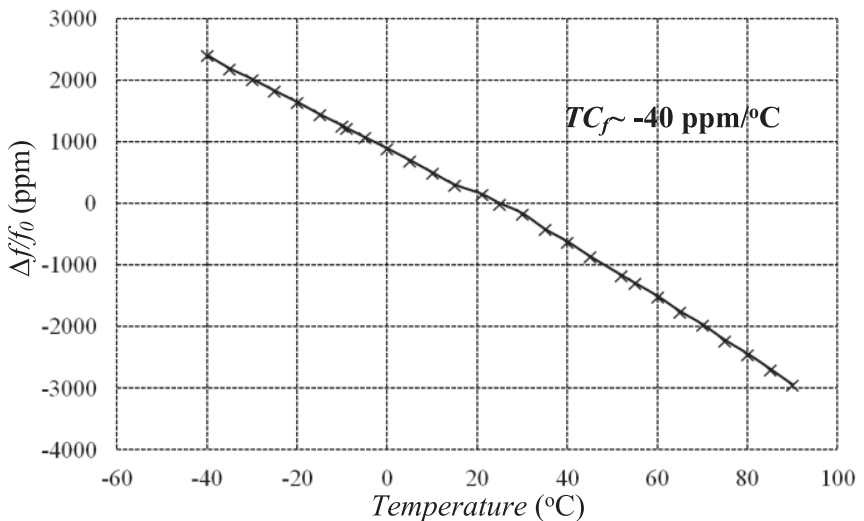

(a)

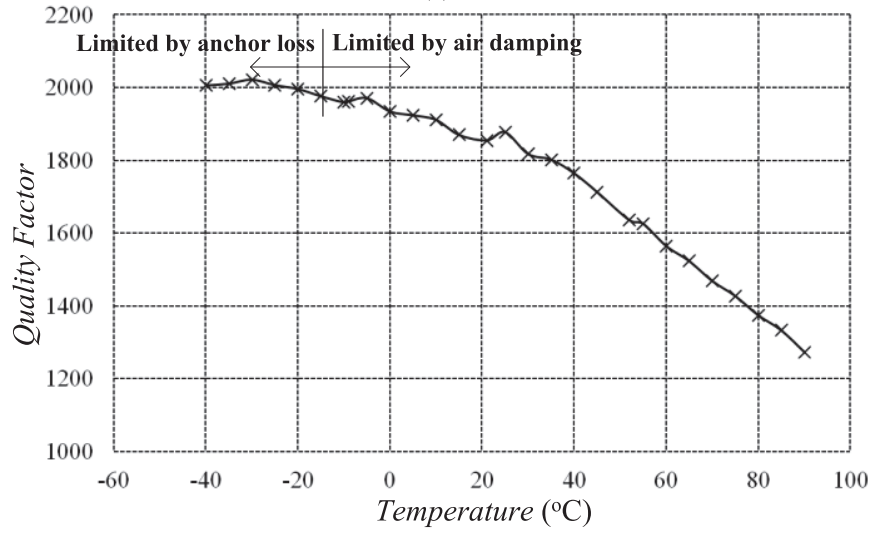

(b)

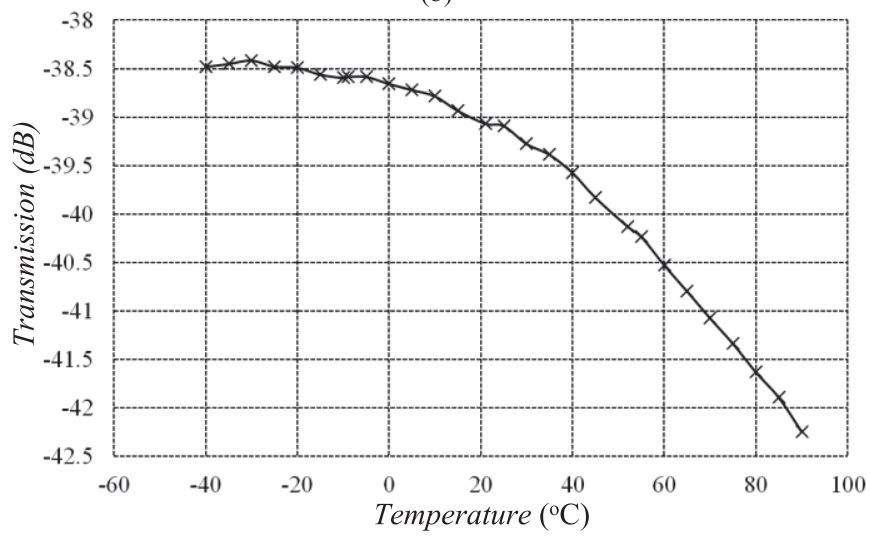

(c)

Fig. 9. Temperature dependence of the resonator's (a) resonance frequency, (b) quality factor, and (c) transmission.

the proposed device could potentially be narrowed by a postprocess timed isotropic etch. Xenon di-fluoride $\left(\mathrm{XeF}_{2}\right)$ vapor is a promising candidate to perform this etch, as it has already been successfully used to release MEMS structures (e.g. [3]) and is highly selective to silicon.

\section{B. Effect of Operating Temperature}

The effect of ambient temperature on the frequency response of the resonator at atmospheric pressure was determined using the single-ended test setup of Fig. 6(a) over the temperature range of -40 to $90{ }^{\circ} \mathrm{C}$. The temperature coefficient of frequency $\left(T C_{f}\right)$ was found to be $\sim-40 \mathrm{ppm} /{ }^{\circ} \mathrm{C}$, 
TABLE II

Comparison Between the Resonator Proposed in THis Work ANd StATE-OF-THE-ART

\begin{tabular}{|c|c|c|c|c|c|c|c|c|c|}
\hline & Type & Mode & $\begin{array}{l}\text { Freq. } \\
\text { (MHz) }\end{array}$ & Quality factor & $\begin{array}{l}\text { Transmission } \\
\text { (dB) }\end{array}$ & $\begin{array}{l}R_{x}^{*} \\
(\Omega)\end{array}$ & $\begin{array}{l}V_{D C} \\
(V)\end{array}$ & $\begin{array}{l}\text { Freq. } \\
\text { tuning }\end{array}$ & Features \\
\hline$[2]$ & Piezo. & $\begin{array}{l}\text { Thin-film } \\
\text { bulk } \\
\text { acoustic }\end{array}$ & $\begin{array}{l}1500 \\
1800\end{array}$ & $\begin{array}{c}350 \\
54\end{array}$ & $>-5$ & $\sim 50$ & - & $x$ & $\begin{array}{l}\text { Zinc oxide }(\mathrm{ZnO}) \text { and lead- } \\
\text { zirconium-titanate } \\
\text { FBARs }\end{array}$ \\
\hline$[5]$ & Piezo. & Contour & 271 & 2100 & - & 188 & - & $x$ & AlN contour mode resonators \\
\hline$[6]$ & Piezo. & Contour & 968 & 1805 & - & 56 & - & $\checkmark$ & $\begin{array}{l}\text { AlN resonators with integrated } \\
\text { heaters for temperature } \\
\text { controlled operation }\end{array}$ \\
\hline$[7]$ & Piezo. & Contour & $\begin{array}{l}181 \\
386 \\
\end{array}$ & $\begin{array}{l}706 \\
172 \\
\end{array}$ & - & $\begin{array}{l}250 \\
3 \mathrm{k} \\
\end{array}$ & - & $x$ & $\begin{array}{l}\text { Reconfigurable AlN resonator } \\
\text { based on integrated switches }\end{array}$ \\
\hline$[8]$ & Piezo. & Lateral & 527.4 & 1780 & - & 110 & - & $\times$ & $\begin{array}{l}\text { Lithium-Niobate }\left(\mathrm{LiNbO}_{3}\right) \\
\text { piezoelectric resonators }\end{array}$ \\
\hline \multirow{2}{*}[3]{} & \multirow{2}{*}{$\begin{array}{l}\text { Cap./ } \\
\text { piezo. }\end{array}$} & \multirow{2}{*}{ Wine-glass } & \multirow{2}{*}{51} & \multicolumn{3}{|c|}{$1 \mu \mathrm{m}$ wide supports } & \multirow{2}{*}{ - } & \multirow{2}{*}{$\times$} & \multirow{2}{*}{$\begin{array}{l}\text { AlN disk resonators with } \\
\text { capacitive-piezoelectric } \\
\text { actuation/ sensing electrodes } \\
\text { above/ below the structure with } \\
260 \mathrm{~nm} \text { gap and anchor beams } \\
\text { of } 1-2 \mu \mathrm{m} \text { width }\end{array}$} \\
\hline & & & & 12,748 & -34 & $4.9 \mathrm{k}$ & & & \\
\hline \multirow{4}{*}{ [4] } & \multirow{4}{*}{$\begin{array}{l}\text { Cap./ } \\
\text { piezo. }\end{array}$} & \multirow{4}{*}{ Radial } & \multirow{4}{*}{300} & \multicolumn{3}{|c|}{$250 \mathrm{~nm}$ gap and $1.2 \mu \mathrm{m}$ anchor } & & \multirow{4}{*}{$\times$} & \multirow{4}{*}{$\begin{array}{l}\text { AlN capacitive-piezoelectric } \\
\text { disk resonators with ON/ OFF } \\
\text { switching capability utilizing } \\
\text { capacitive electrodes above } \\
\text { below the structure with } 120- \\
250 \mathrm{~nm} \text { gap and center anchors }\end{array}$} \\
\hline & & & & 8,800 (vacuum) & -40 & $9.9 \mathrm{k}$ & & & \\
\hline & & & & \multicolumn{3}{|c|}{$120 \mathrm{~nm}$ gap and $1.8 \mu \mathrm{m}$ anchor } & & & \\
\hline & & & & 6,600 (vacuum) & -30 & $3.01 \mathrm{k}$ & & & \\
\hline [9] & Cap. & $\begin{array}{l}1^{\text {ary }} \text { elliptic } \\
2^{\text {ary }} \text { elliptic }\end{array}$ & $\begin{array}{l}2.9 \\
5.9\end{array}$ & $\begin{array}{c}66,000 \text { (1 mTorr }) \\
100,000 \text { (1 mTorr })\end{array}$ & $\begin{array}{l}-53 \\
-31\end{array}$ & $\begin{array}{l}44.6 \mathrm{k} \\
3.4 \mathrm{k}\end{array}$ & 10 & $\checkmark$ & $\begin{array}{l}\text { Bulk-mode disk gyros in } 111 \\
\text { and } 100 \text { single-crystalline Si } \\
\text { with } 180-250 \mathrm{~nm} \text { capacitive } \\
\text { gaps }\end{array}$ \\
\hline [10] & Cap. & Wine-glass & $\begin{array}{c}210 \\
1520\end{array}$ & $\begin{array}{l}7,700 \text { (200 } \mu \text { Torr }) \\
3,000 \text { (200 } \mu \text { Torr })\end{array}$ & - & $\begin{array}{l}9.16 \mathrm{k} \\
791.6 \mathrm{k}\end{array}$ & $\begin{array}{c}15 \\
5\end{array}$ & $\checkmark$ & $\begin{array}{l}\text { Poly-Si wine-glass mode ring } \\
\text { resonators with } 63-85 \mathrm{~nm} \\
\text { capacitive gaps and } 1.6 \mu \mathrm{m} \text { wide } \\
\text { anchor beams }\end{array}$ \\
\hline$[17-18]$ & Cap. & $2^{\text {ary }}$ elliptic & 1.5 & 33,000 (10 mTorr) & -50 & $31.5 \mathrm{k}$ & 50 & $\checkmark$ & $\begin{array}{l}\text { Dodecagon disk gyro with } \\
\text { combs utilizing a } \mathrm{Si} \text { structural } \\
\text { layer }\end{array}$ \\
\hline & & & & $10 \mu \mathrm{m}$ & de supports & & & & $\mathrm{Si}$ wine-glass disk resonators \\
\hline $\begin{array}{l}\text { This } \\
\text { work }\end{array}$ & Piezo & Wine-glass & 14.63 & $\begin{array}{c}4,894 * *(\leq 100 \mathrm{mTorr}) \\
1,952 \text { (atm.) }\end{array}$ & -22 & $1.2 \mathrm{k}$ & - & $\checkmark$ & $\begin{array}{l}\text { sensing, electrostatic frequency } \\
\text { tuning, and anchor beams of } \\
10 \mu \mathrm{m} \text { width }\end{array}$ \\
\hline
\end{tabular}

*The motional resistance $R_{x}$ is estimated based on $R_{x}=100\left(1 / T_{\max }-1\right)$, if not reported, where $T_{\max }$ is the transmission at resonance. This assumes a measurement characteristic impedance of $50 \Omega$.

**The quality factor of the device is limited by the $10 \mu \mathrm{m}$ support width imposed by the fabrication technology. It could be improved by using another technology which allows for smaller support widths in order to reduce the anchor loss as shown in [3].

as a consequence mainly of thermal expansion of the structure. The $T C_{f}$ is slightly higher than devices presented in the literature (e.g., [10], [12]), which is probably due to the differences between the properties of the structural materials used. Figure 9 presents the effects of temperature on the resonance frequency, the quality factor, and the transmission of the resonator. The device's quality factor is limited by two main damping mechanisms, namely, anchor loss due to the supports, and air damping resulting from the squeeze film damping between the resonating disk structure and the electrodes. At low temperatures, the anchor loss is the dominant mechanism, and therefore the quality factor 


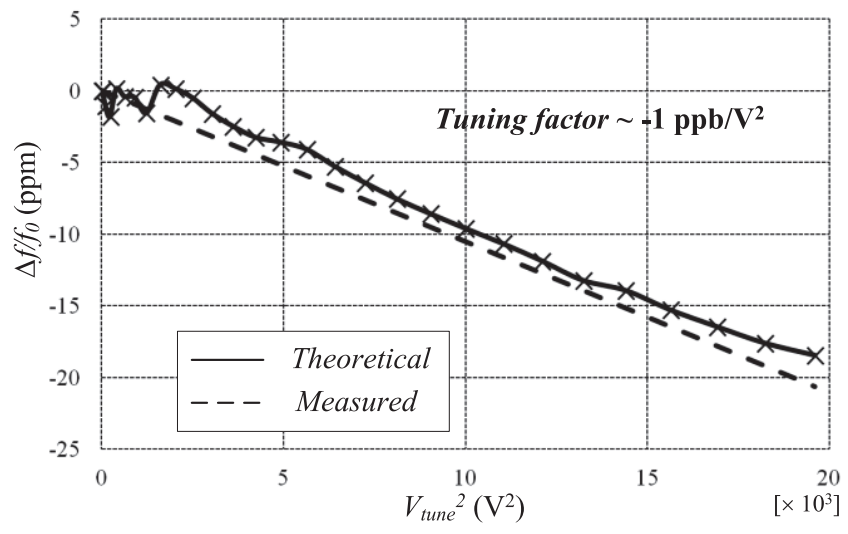

Fig. 10. Fractional change in the resonance frequency vs (tuning voltage $)^{2}$.

is constant. As the temperature increases, the air damping effect increases due to the increase of the air viscosity [24], [25], as illustrated in Fig. 9(b). Expectedly, the transmission curve follows the same pattern of the quality factor curve as shown in Fig. 9(c).

\section{Electrostatic Tuning}

The electrostatic tuning capability of the resonator was characterized using the single-ended test setup of Fig. 6(a), with an external DC source used to set the tuning voltage. The ground nodes of the source and network analyzer were connected together to ensure a common reference voltage between the ground plane of the disk and the applied tuning voltage, and thus ensure the intended voltage difference between the tuning electrodes and the disk. Figure 10 presents the measured fractional change in the resonance frequency with respect to the tuning voltage applied, along with the theoretical variation expected based on (8). The device exhibits a tuning factor of $\sim-1 \mathrm{ppb} / \mathrm{V}^{2}$ that could serve to compensate for minor variations in operating conditions (e.g., temperature) or for implementing tunable high frequency synthesizers, as in [1], where a frequency multiplier could be used to increase the absolute frequency change. Notably, no change in the resonator quality factor was observed throughout the tuning voltage range tested.

\section{DISCUSSION}

Table II compares the device proposed here and implemented in a low-cost commercial MEMS technology to other state-of-the-art bulk-mode resonators. The table highlights its reasonable quality factor, limited by the anchor loss dictated by the technology, and its superior motional resistance. The device achieves the lowest signal loss and motional resistance amongst the bulk-mode devices in comparison. The achieved motional resistance is a key advantage for the device presented in this work, as it results in significant simplification of the associated electronics for realizing complete systems (e.g., oscillator and phase locked loop in [1]) resulting in lower power consumption and phase noise. The performance demonstrated here is achieved without the need for submicron gaps, as in [3], [4], and [9]-[13], or high DC polarization voltages, as in [14]-[18]. In fact, the device requires no DC voltage for its operation. The Q-factor can be further improved by decreasing the width of the suspension beams to reduce anchor loss, either by post-processing as explained in section IV.A, or by using another technology with smaller critical dimensions. Compared to other piezoelectric disk resonators (e.g., [3], [4]), the proposed design adds tuning functionality to piezoelectric resonators by employing sep-arate electrostatic electrodes. Although the tuning range of the proposed design is relatively low due to the large gap imposed by the fabrication technology, it could be significantly improved by using a technology with narrower lateral gaps. The structure also allows for various frequency devices to be fabricated concurrently, unlike FBARs. It is worth mentioning that the present structure is achieved in a relatively simple commercial MEMS technology, and that its performance could be enhanced by implementation in a technology with smaller critical dimensions.

\section{CONCLUSION}

This work demonstrates the first use of transverse piezoelectric actuation to excite a bulk-mode silicon resonator in the wine-glass mode to offer a desirable combination of reasonably high quality factor and frequency, along with the lowest motional resistance amongst comparable disk resonators without requiring any DC voltage for operation. The device of this work is also the first demonstrated wineglass bulk-mode disk resonator built using a commercially available low-cost MEMS fabrication process. The device is measured to have a resonance frequency of $\sim 15 \mathrm{MHz}$ and, a quality factor of $\sim 2,000$ at atmospheric pressure improving to $\sim 5,000$ in a 100 mTorr vacuum, while exhibiting a temperature coefficient of $\sim-40 \mathrm{ppm} /{ }^{\circ} \mathrm{C}$. External capacitive electrodes were also successfully used to optionally adjust the frequency of resonance through DC voltage tuning and thus combine the advantages of both electrostatic and piezoelectric actuation.

\section{ACKNOWLEDGMENT}

The authors would like to thank CMC Microsystems for providing the layout design tools and the testing equipment needed.

\section{REFERENCES}

[1] F. Nabki, K. Allidina, F. Ahmad, P.-V. Cicek, and M. N. El-Gamal, "A highly integrated $1.8 \mathrm{GHz}$ frequency synthesizer based on a MEMS resonator," IEEE J. Solid-State Circuits, vol. 44, no. 8, pp. 2154-2168, Aug. 2009.

[2] Q.-X. Su, P. Kirby, E. Komuro, M. Imura, Q. Zhang, and R. Whatmore, "Thin-film bulk acoustic resonators and filters using $\mathrm{ZnO}$ and leadzirconium-titanate thin films," IEEE Trans. Microw. Theory Techn., vol. 49, no. 4, pp. 769-778, Apr. 2001.

[3] L.-W. Hung and C. T.-C. Nguyen, "Capacitive-piezoelectric AlN resonators with Q>12,000," in Proc. IEEE Int. Conf. Micro Electro Mech. Syst., Jan. 2011, pp. 173-176.

[4] R. A. Schneider and C. T.-C. Nguyen, "On/off switchable high-Q capacitive-piezoelectric AlN resonators," in Proc. IEEE Int. Conf. Micro Electro Mech. Syst., Jan. 2014, pp. 1265-1268.é 
[5] C. Zuo, N. Sinha, and G. Piazza, "Very high frequency channel-select MEMS filters based on self-coupled piezoelectric AlN contour-mode resonators," Sens. Actuators A, Phys., vol. 160, pp. 132-140, May 2010.

[6] M. Rinaldi, Y. Hui, C. Zuniga, A. Tazzoli, and G. Piazza, "High frequency AlN MEMS resonators with integrated nano hot plate for temperature controlled operation," in Proc. IEEE Int. Freq. Control Symp., May 2012, pp. 1-5.

[7] G. Hummel, Y. Hui, and M. Rinaldi, "Highly reconfigurable aluminum nitride MEMS resonator using 12 monolithically integrated phase change material switches," in Proc. IEEE Int. Conf. Solid-State Sens., Actuators, Microsyst. (TRANSDUCERS), Jun. 2015, pp. 323-326.

[8] S. Gong and G. Piazza, "Design and analysis of lithium-niobate-based high electromechanical coupling RF-MEMS resonators for wideband filtering," IEEE Trans. Microw. Theory Techn., vol. 61, no. 1, pp. 403-414, Jan. 2013.

[9] H. Johari and F. Ayazi, "High-frequency capacitive disk gyroscopes in (100) and (111) silicon," in Proc. IEEE Int. Conf. Micro Electro Mech. Syst. (MEMS), Jan. 2007, pp. 47-50.

[10] Y. Xie, S.-S. Li, Y.-W. Lin, Z. Ren, and C. T.-C. Nguyen, "1.52-GHz micromechanical extensional wine-glass mode ring resonators," IEEE Trans. Ultrason., Ferroelectr., Freq. Control, vol. 55, no. 4, pp. 890-907, Apr. 2008

[11] J. R. Clark, W.-T. Hsu, M. A. Abdelmoneum, and C. T.-C. Nguyen, "High-Q UHF micromechanical radial-contour mode disk resonators," J. Microelectromech. Syst., vol. 14, no. 6, pp. 1298-1310, Dec. 2005.

[12] J. Wang, Z. Ren, and C. T.-C. Nguyen, "1.156-GHz self-aligned vibrating micromechanical disk resonator," IEEE Trans. Ultrason. Ferroelectr, Freq. Control, vol. 51, no. 12, pp. 1607-1628, Dec. 2004

[13] Y.-W. Lin, S. Lee, S.-S. Li, Y. Xie, Z. Ren, and C. T.-C. Nguyen, "Seriesresonant VHF micromechanical resonator reference oscillators," IEEE J. Solid-State Circuits, vol. 39, no. 12, pp. 2477-2491, Dec. 2004

[14] L. Khine, M. Palaniapan, and W.-K. Wong, "6Mhz bulk-mode resonator with Q values exceeding one million," in Proc. IEEE Int. Conf. Solid-State Sens., Actuators, Microsyst., Jun. 2007, pp. 2445-2448.

[15] J. E.-Y. Lee and A. A. Seshia, "5.4-MHz single-crystal silicon wine glass mode disk resonator with quality factor of 2 million," Sens. Actuators A, Phys., vol. 156, pp. 28-35, Nov. 2009.

[16] M. Elsayed, F. Nabki, and M. El-Gamal, "A 2000\%s dynamic range bulk mode dodecagon gyro for a commercial SOI technology," in Proc. IEEE Int. Conf. Electron., Circuits, Syst., Dec. 2011, pp. 264-267.

[17] M. Y. Elsayed, F. Nabki, and M. N. El-Gamal, "A combined comb/bulk mode gyroscope structure for enhanced sensitivity," in Proc. IEEE Int. Conf. Micro Electro Mech. Syst. (MEMS), Jan. 2013, pp. 649-652.

[18] M. Y. Elsayed, F. Nabki, and M. N. El-Gamal, "A novel comb architecture for enhancing the sensitivity of bulk mode gyroscopes," Sensors, vol. 13, pp. 16641-16656, Dec. 2013.

[19] D. E. Serrano, R. Tabrizian, and F. Ayazi, "Electrostatically tunable piezoelectric-on-silicon micromechanical resonator for real-time clock," IEEE Trans. Ultrason., Ferroelectr., Freq. Control, vol. 59, no. 3 , pp. 358-365, Mar. 2012.

[20] M. Hodjat-Shamami, A. Norouzpour-Shirazi, R. Tabrizian, and F. Ayazi, "A dynamically mode-matched piezoelectrically transduced highfrequency flexural disk gyroscope," in Proc. IEEE Int. Conf. Micro Electro Mech. Syst. (MEMS), Jan. 2015, pp. 789-792.

[21] S. D. Senturia, Microsystem Design. New York, NY, USA: Springer, 2001, p. 134.

[22] C. Acar and A. Shkel, "Electrical design of MEMS gyroscopes," in MEMS Vibratory Gyroscopes. New York, NY, USA: Springer, Sep. 2009 , pp. 1-29.

[23] PiezoMUMPs Design Handbook, Revision 1.3, MEMSCAP, Durham, NC, USA, 2014

[24] E. Hosseinian, P.-O. Theillet, and O. N. Pierron, "Temperature and humidity effects on the quality factor of a silicon lateral rotary microresonator in atmospheric air," Sens. Actuators A, Phys., vol. 189, pp. 380-389, Jan. 2013.

[25] P. T. Tsilingiris, "Thermophysical and transport properties of humid air at temperature range between 0 and $100{ }^{\circ} \mathrm{C}$," Energy Convers. Manage., vol. 49, pp. 1098-1110, May 2008.é

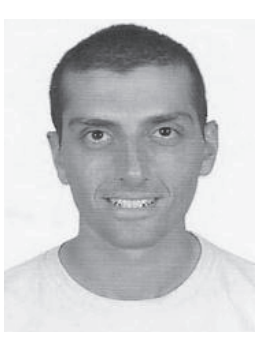

Mohannad Y. Elsayed (S'08) was born in Cairo, Egypt, in 1985. He received the B.Sc. (Hons.) degree and the M.Sc. degree in electronics and communications engineering from Cairo University, Giza, Egypt, in 2007 and 2010, respectively. He is currently pursuing the Ph.D. degree with the Wireless Integrated Circuits and MEMS Laboratory, McGill University, Montreal, QC, Canada.

He was a Teaching Assistant with the Department of Electronics and Communications Engineering, Cairo University, and a Research Assistant with the Yousef Jameel Science and Technology Research Center, American University in Cairo, Cairo. He has held internship positions with several institutions, including the Rice Automated Nanoscale Design Laboratory, Rice University, Houston, TX, and Thales, Canada. He authored or co-authored 11 journal and conference papers. He holds three patents in novel circuit designs and MEMS devices. His research interests include MEMS devices and analog circuits, with a special focus on MEMS sensors and resonators, and the monolithic integration of MEMS with CMOS integrated circuits.

Mr. Elsayed has held scholarships from McGill University and the Microsystems Strategic Alliance of Quebec (ReSMiQ).

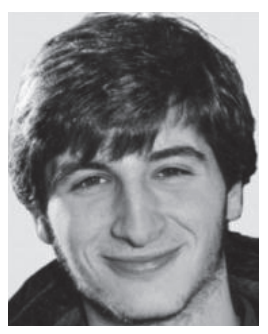

Paul-Vahé Cicek (S'09-M'14) received the B.Eng. (Hons.) degree in electrical engineering (minor in arts) from McGill University, Montreal, QC, Canada, in 2006, where he is currently pursuing the $\mathrm{Ph} . \mathrm{D}$. degree in electrical engineering.

He interned with the McGill RFIC Group, from 2005 to 2006, developing a novel wafer-level packaging technology. From 2006 to 2007, and from 2009 to 2011, he was a Research Assistant with the Wireless ICs and MEMS Laboratory, where he was involved in the development of MEMS technologies and devices. In 2014, he was selected to become an Assistant Professor with the Department of Computer Science within the Microelectronics Engineering Program, Université du Québec à Montréal, where he will shortly begin to assume his functions. His research interests include novelty MEMS technologies and devices, monolithic integration of MEMS and VLSI electronics, advanced packaging and encapsulation, and microfluidic systems. He has contributed to 13 publications, and holds two issued patents and five pending patents.

Mr. Cicek has received funding from the Quebec Fund for Research in Nature and Technology, the Microsystems Strategic Alliance of Quebec (ReSMiQ), the Natural Sciences and Engineering Research Council of Canada, and McGill University.

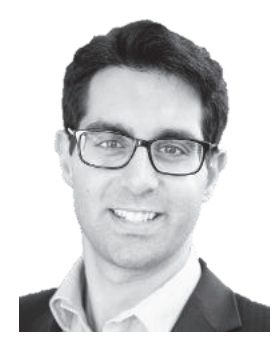

Frederic Nabki (S'99-M'10) received the B.Eng. (Hons.) degree in electrical engineering and the Ph.D. degree in electrical engineering from McGill University, Montreal, QC, Canada, in 2003 and 2010, respectively.

$\mathrm{He}$ joined the Université du Québec à Montréal (UQAM), Montreal, in 2008, where he is currently an Associate Professor of Microelectronics Engineering. His research interests include microelectromechanical systems (MEMS) and $\mathrm{RF} /$ analog microelectronics, specifically focusing on the creation of next-generation MEMS processes using advanced materials, the integration of MEMS devices with CMOS systems, the modeling of MEMS devices, and the design of CMOS phase-locked loops, ultra-wideband transceivers, and MEMS interface circuits. He has authored two book chapters and over sixty publications, and holds five issued patents and nine pending patent applications related to MEMS and CMOS/MEMS monolithic integration.

Dr. Nabki is a Member of the Quebec Order of Engineers and serves as the Treasurer of the Montreal Section of the IEEE. He is a Founder and the Associate Director of the Institutional Research Centre on the Co-design and Fabrication of Microsystems with UQAM, and jointly manages the Microtechnology and Microsystems Laboratory. He was a recipient of the Governor General of Canada's Academic Bronze Medal and the UQAM Faculty of Science Early Career Research Award. He holds or has held financial support from the Microsystems Strategic Alliance of Quebec (ReSMiQ), the Quebec Fund for Research in Nature and Technology, the Natural Sciences and Engineering Research Council of Canada, and the Canada Foundation for Innovation. 


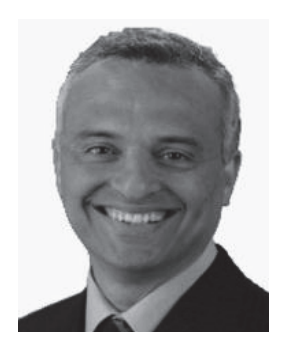

Mourad N. El-Gamal received the B.Sc. (Hons.) degree in electrical engineering from Ain-Shams University, Cairo, Egypt, in 1987; the M.Sc. degree (minor in computer science) in electrical engineering from Vanderbilt University, Nashville, TN, in 1993; and the Ph.D. degree in electrical engineering from McGill University, Montreal, QC, Canada, in 1998.

He has held the William Dawson Scholar Chair with the Department of Electrical and Computer Engineering, McGill University, since 2004, and served as a Member of the Steering Committee of the Nanofabrication Facility of the Institute for Advanced Materials. He was an Associate Vice Principal-Research and International Relations (VP-RIR) with McGill University, from 2007 to July 2008. Among the main initiatives that he spearheaded was the development of a comprehensive university-industry partnership strategic plan, including all aspects of technology transfer and commercialization. In 2002, while on leave from McGill University, he was the Director of Engineering, and then the Vice President and General
Manager of the Wireless Business Unit of MEMSCAP, France-a 165-employee publicly traded company specializing in microelectromechanical systems (MEMS). Earlier, he was with IBM Research and the French telecommunications company, ALCATEL. He consults for companies and the Canadian Government on policies and strategies. His research focus has been on the combination of these technologies to produce new knowledge and innovative solutions for a wide range of applications, including wireless communications systems, health care, and aerospace. He has authored over 80 technical articles and three book chapters, and his team has received several research awards. He holds one patent and currently has five patents filed in novel micro/nano processes and devices. His research interests are in micro and nanoscale integrated electronics and mechanical systems, with a special interest in advanced materials.

Dr. El-Gamal has been a recipient of numerous teaching awards. He was an Associate Editor of the IEEE TRANSACTIONS ON CIRCUITS AND SYSTEMS-I from 2006 to 2008, and is currently the Chair of the Biomedical, Sensors, Displays, and MEMS Subcommittee of the IEEE Custom Integrated Circuits Conference. 